\title{
Atualização em avaliação psicológica e neuropsicológica de crianças e adolescentes
}

\author{
Nayane Martoni Piovezan ${ }^{-}$- Universidade São Francisco, Itatiba, Brasil
}

Hutz, C. S. (Org.). (2010). Avanços em Avaliação Psicológica e Neuropsicológica de crianças e adolescentes. São Paulo: Casa do Psicólogo, 373 p.

O avanço da área de Avaliação Psicológica tem sido constatado pelo crescente número de profissionais qualificados e de publicações que aumentam a amplitude de materiais importantes para consulta e necessários para seu crescimento. A despeito disso, ainda há um longo caminho a se percorrer para que os profissionais e estudiosos da área possam realizar avaliações e diagnósticos dos diversos fenômenos psicológicos por meio de instrumentos válidos e precisos. A organização deste livro, feita por Claudio Simon Hutz, pretendeu contribuir na redução das dificuldades percebidas por meio de sua própria experiência tanto na área da pesquisa quanto na prática de avaliação e diagnóstico.

O livro é organizado em 13 capítulos que abarcam os diversos contextos em que a avaliação psicológica pode ser aplicada. O primeiro, de autoria de Luciana Karine de Souza e intitulado "Avaliação da amizade na infância: da troca de papéis à melhor amizade" trata do desenvolvimento social, mais especificamente da habilidade sociocognitiva de troca de papéis na infância baseando-se na perspectiva de Robert Selman, que considerou os pressupostos de Jean Piaget para aprofundar essa questão. A troca de papel é tida como imprescindível para o estabelecimento de amizades recíprocas, duradouras e próximas. Além de tratar dos aspectos teóricos relativos ao tema, a autora expõe também aspectos das habilidades sociais, visto que uma criança socialmente habilidosa faz amizades e as mantém. Ao longo desse capítulo, são feitas referências a alguns instrumentos de medida e técnicas relacionados aos dois temas.

No segundo capítulo, "Avaliação cognitiva de crianças e jovens: aspectos multidimensionais", Solange M. Weschsler, Tatiana de Cássia Nakano, Maiana Faria O. Nunes e Carla Alexandra da S. M. Minervino discutem sobre a necessidade de uma visão multidimensional na avaliação da inteligência, criatividade e de leitura e escrita de crianças. As autoras expõem a conceituação e modelos teóricos da inteligência, de um instrumento de avaliação, a Bateria de Habilidade Cognitivas Woodcock-Johnson III e as limitações das medidas de inteligência. Em seguida, a

1 nanny_mp@yahoo.com.br criatividade é tratada em termos de avaliação e suas dimensões e procedimentos e também é focalizado um instrumento, o Teste Brasileiro de Criatividade Figurativa Infantil, para, então, versarem sobre a relação entre a inteligência e a criatividade. Por fim, finalizam a perspectiva multidimensional da avaliação cognitiva infantil falando da avaliação da leitura e escrita, especificamente do Inventário de Identificação de Sinais Disléxicos, demonstrando a importância da coleta de informações advindas de diversas fontes e dimensões cognitivas.

Já na perspectiva do adolescente, Daniela Sacramento Zanini e Helenides Mendonça escreveram terceiro capítulo, intitulado "Avaliação das estratégias de coping em adolescentes brasileiros". É descrito um estudo que teve por objetivo discutir a aplicabilidade do conceito de coping e adaptação de um instrumento para avaliação desse construto aplicado a adolescentes goianos, o Coping Response Inventory - Youth Form (CRIYouth). São apresentados no tópico de resultados, as estatísticas descritivas, consistência interna e dimensionalidade do instrumento. A discussão e conclusão guiam o leitor à dar atenção também aos aspectos culturais envolvidos nas estratégias de enfrentamento a problemas, assim como no estabelecimento de relações entre essas variações culturais à luz dos problemas específicos expostos pelos adolescentes.

A "Avaliação de problemas de comportamento infantil através do Child Behavior Checklist (CBCL)" é o foco do capítulo escrito por Denise R. Bandeira. Juliane Callegaro Borsa, Joice Dickel Segabinazi e Adriane X. Arteche. O instrumento, que também pode ser nomeado por Lista de Verificação Comportamental para crianças, é descrito em termos de suas características estruturais, aplicação, estudos realizados, suas características psicométricas e adaptação e validação do CBCL para o Brasil. Mais especificamente, as autoras versam sobre a aplicação desse instrumento no Centro de Avaliação Psicológica da Universidade Federal do Rio Grande do Sul (CAP UFRGS). Assim, o capítulo fornece informações importantes e atuais acerca dos estudos e das evidências de validade e fidedignidade para o CBCL integradas por eles.

O quinto capítulo, escrito por André Luiz Moraes, Denise Balem Yates, Aline Sokolovsky e 
Clarissa Marceli Trentini, refere-se à "Inteligência versus funções executivas em crianças e adolescentes: um estudo correlacional". São descritos os aspectos teóricos principais e o estudo realizado que teve por objetivo verificar a associação entre funções executivas e inteligência. Foram utilizados os dados de um estudo de normatização para o Teste Wiscosin de Classificação de Cartas (WCST) para o Brasil. Além desse instrumento, foram coletadas informações por meio, também, de um questionário sócio-demográfico, do Child Behavior Checklist, Teacher's Report Form, Subteste Cubos e Subteste Vocabulário, ambos pertencentes à Escala Wechsler de Inteligência (WISCIII). Os autores descrevem os resultados em forma de estatísticas descritiva e inferencial e apresentam a conclusão do estudo de correlação.

Janaína T. B. Pacheco, Marúcia Patta Bardagi, Caroline Tozzi Repold e Claudio Simon Hutz são os autores do sexto capítulo, que versa sobre "Instrumentos de avaliação das práticas educativas parentais no contexto brasileiro". Há grande e crescente interesse em estudar o impacto das práticas educativas familiares no desenvolvimento dos membros de uma família. Assim, nesse capítulo é definido o conceito de práticas parentais, bem como suas variáveis mediadoras, os instrumentos e outras estratégias de avaliação. O Inventário de Estilos Parentais (IEP), o Roteiro de Entrevista de Habilidades Sociais Educativas Parentais (RE-HSE-P), o Relatório de Pais, a Escala de Práticas Parentais e o Inventário de Práticas Parentais são alguns dos instrumentos apresentados pelos autores. A influência das práticas educativas dos pais torna-se importante de se detectar levando em conta o fato de as crianças e os adolescentes estarem frequentemente expostos a diferentes tipos de influências externas, como por exemplo, do grupo de pares, da escola e da mídia e de suas conseqüências no desenvolvimento de cada indivíduo.

Já no que se refere à avaliação da compreensão de linguagem oral, Maria Cristina R. A. Joly, Caroline Tozzi Repold e Anelise Silva Dias, são as autoras do sétimo capítulo, que trata da "Avaliação da linguagem oral de crianças paulistas e gaúchas pela Bateria Informatizada de Linguagem Oral (BILOv2)". É descrito um estudo com a BILO, além de versarem sobre a linguagem e a escolarização e dos estudos já realizados com esse instrumento de medida que evidenciam sua validade. O estudo foi realizado com crianças de São Paulo e Rio Grande do Sul e seus resultados são apresentados por meio de análises estatísticas descritivas e inferenciais, considerando algumas variáveis de caracterização dos participantes, como região onde mora, gênero, idade e série escolar e verificação do índice de precisão e evidências de validade para a BILO. Dada a importância da linguagem no contexto educacional, um instrumento que possibilite a avaliação da compreensão da linguagem oral é de relevância.

As "Tarefas de memória implícita" são o foco do capítulo escrito por Jerusa Fumagalli de Salles, Candice Steffen Holderbaum, Denise Bernardi e Rodrigo S. Kreitchmann. Os autores discorrem sobre a dificuldade de se avaliar a memória implícita sem que haja interferência da memória explícita e tratam de alternativas disponíveis na literatura nacional e internacional para minimizar essa dificuldade. Expõem o conceito e os tipos de memória implícita, que são formas simples de memória não consciente, como por exemplo a memória procedimental, aprendizado perceptual, aprendizado emocional, mas versam com maior profundidade e especificidade sobre o priming semântico. Desse ponto, falam das tarefas de identificação perceptual, de completar fragmentos, tarefas de julgamento e sobre o paradigma de priming semântico, abarcando também os estudos nacionais dessa natureza.

Sobre os requisitos dos testes psicológicos e da amostra de estudos empíricos, Claudette M. M. Vendramini e Solange M. Wechsler assinam o capítulo nove, intitulado "Considerações teóricas sobre a representatividade das amostras de normatização de testes psicológicos". Ao longo do texto, são tratados assuntos como o processo de construção de instrumentos psicológicos, as características e critérios psicométricos que um teste deve possuir, da escolha e qualidade dos testes utilizados num processo de avaliação psicológica e finalmente da representatividade das amostras. Levando em conta as características da amostra, falam também sobre a significância e o poder dos testes estatísticos e o desafio de normatizar (obter normas para comparação dos resultados) os testes brasileiros.

"Avaliação neuropsicológica das funções executivas na infância e adolescência" é o décimo capítulo desse livro, que foi escrito por Alessandra Gotuzo Seabra, Natália Martins Dias e Bruna Tonietti Trevisan e discorre sobre a conceituação das funções executivas (FE), as especificidades anatômicas cerebrais relativas a essas funções, bem como seus processos adjacentes, como a memória de trabalho, atenção, inibição e flexibilidade cognitivas. O conteúdo do texto perpassa especialmente por testes psicológicos para avaliação das FE em crianças e adolescentes, embora haja carência de instrumentos com esse fim e esse fato comprometa o desenvolvimento e ampliação de pesquisas nessa área e ressalta a importância de avaliar as FE para que seja melhor compreendido o 
desenvolvimento normal e também entendimento de possíveis comprometimentos para que intervenções sejam delineadas de modo eficaz.

O décimo primeiro capítulo, de autoria de Maycoln Teodoro, Mirian B. da S. Escanhuela, Adriana R. Binsfeld e Bruna Mônego, relata uma pesquisa sobre a "Avaliação das relações familiares e depressão em crianças e adolescentes institucionalizados" e discorre sobre a importância das relações familiares e suas conseqüências para o bem estar e qualidade de vida infantis, por exemplo. Foram utilizados uma entrevista estruturada, o Familiograma e o Inventário de Depressão Infantil (CDI). Os resultados do estudo descrevem a afetividade e o conflito familiar pelo prisma das crianças e adolescentes que viviam com a familia e na instituição.

O penúltimo capítulo trata das "Dificuldades para decisão profissional e traços de personalidade em adolescentes", no qual Maiana F. O. Nunes, Ana Paula P. Noronha, Carlos H. S. S. Nunes e Ricardo Primi discorrem sobre o processo de Orientação Profissional, assim como as dificuldades permeadas ao processo de decisão e escolha de uma profissão e suas subcategorias. Aliado a isso, versam sobre os traços de personalidade e a carência de estudos que os relacionem ao processo de tomada de decisão para escolha profissional. Assim, descrevem um estudo realizado com adolescentes que responderam $\mathrm{O}$ Inventário de Dificuldades de Decisão Profissional (IDDP) e a Bateria Fatorial de Personalidade (BFP). Por meio de análises estatísticas descritivas e inferenciais, os resultados são apresentados considerando as variáveis dos de caracterização dos participantes, as dimensões aferidas pelo IDDP e os fatores de personalidade subjacentes à BFP.

As funções executivas são, também, foco do capítulo escrito por Rochele Paz Fonseca, Camila R. de Oliveira, Gigiane Gindri, Nicolle Zimmermann, Caroline T. Reppold, Maria Alice de Mattos P. Parente. "Teste Hayling: um instrumento de avaliação de componentes das funções executivas" trata do desenvolvimento e descrição do referido teste, bem como da teoria na qual foi baseado, a teoria do Sistema Atencional Supervisor (SAS). É tratado nesse capítulo, também, sobre o processo de adaptação desse instrumento para a realidade brasileira, sua aplicação, registro e atribuição de escores e interpretação.

Este livro, que é um produto do Grupo de Trabalho (GT) sobre Avaliação Psicológica e Neuropsicológica de crianças e adolescentes, mostra o quanto essa área tem crescido e se desenvolvido com a contribuição de profissionais qualificados. Esse material é de relevância para os estudantes, graduados e pós-graduados da Psicologia e certamente contribui para o aprofundamento dos conhecimentos dos psicólogos inseridos nas diversas áreas, pois, além de trazer informações de instrumentos para aferir variados construtos, mostra a importância da avaliação psicológica nos diversos contextos possíveis de atuação dos psicólogos. 